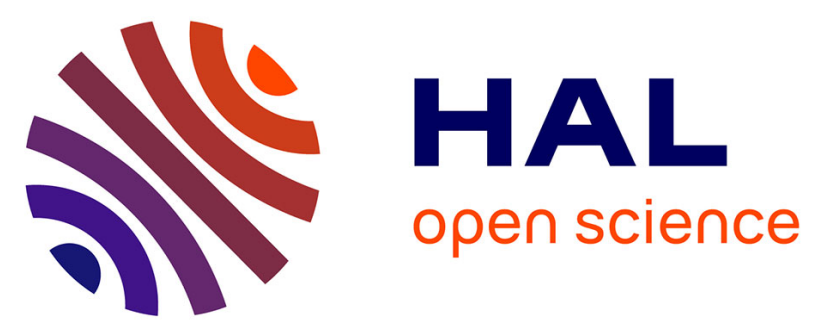

\title{
Libre accès et frais de publication associés (Articles Processing Charges) : une étude de cas de la production scientifique des établissements d'enseignement supérieur et de recherche orléanais
}

\author{
Christophe Boudry, Nathalie Pothier, Manuel Durand-Barthez
}

\section{To cite this version:}

Christophe Boudry, Nathalie Pothier, Manuel Durand-Barthez. Libre accès et frais de publication associés (Articles Processing Charges) : une étude de cas de la production scientifique des établissements d'enseignement supérieur et de recherche orléanais. Ethics, Medicine and Public Health, 2019, 10, pp.65-75. 10.1016/j.jemep.2019.05.005 . hal-02321650

\section{HAL Id: hal-02321650 \\ https://hal.science/hal-02321650}

Submitted on 21 Oct 2019

HAL is a multi-disciplinary open access archive for the deposit and dissemination of scientific research documents, whether they are published or not. The documents may come from teaching and research institutions in France or abroad, or from public or private research centers.
L'archive ouverte pluridisciplinaire HAL, est destinée au dépôt et à la diffusion de documents scientifiques de niveau recherche, publiés ou non, émanant des établissements d'enseignement et de recherche français ou étrangers, des laboratoires publics ou privés. 


\section{Page de titre}

\section{Titre}

«Libre accès et frais de publication associés (Articles Processing Charges) : une étude de cas de la production scientifique des établissements d'enseignement supérieur et de recherche orléanais »

Anglais :

«Open Access and related publication costs: A case study of the scientific output of Higher education and Research institutions of Orléans »

\section{Titre court}

Français :

«Libre accès et frais de publication»

Anglais :

«Open Access and publication costs»

\section{Auteurs}

C. Boudry ${ }^{\mathrm{a}, \mathrm{b}, \mathrm{c}}$, N. Pothier ${ }^{\mathrm{d}}$, M. Durand-Barthez ${ }^{\mathrm{b}, \mathrm{c}}$

\section{Affiliations}

${ }^{a}$ Normandie Univ, UNICAEN, Média Normandie. Esplanade de la Paix. 14032 Caen Cedex 5, France

${ }^{\mathrm{b}}$ Unité régionale de formation à l'information scientifique et technique (URFIST), Ecole Nationale des Chartes, PSL Research University. 17, rue des Bernardins, 75005 Paris, France

c Laboratoire "Dispositifs d'Information et de Communication à l'Ėre Numérique" (DICEN), EA7339, Conservatoire National des Arts et Métiers, 292 rue Saint Martin 75003 Paris, France

d Observatoire des Sciences de l'Univers en région Centre, UMS 3116, Université d'Orléans, CNRS, 1A rue de la Férollerie, 45071 Orléans, France

\footnotetext{
* Auteur correspondant
} 


\section{Adresse email}

christophe.boudry@chartes.psl.eu (Christophe Boudry)

\section{Déclaration de liens d’intérêts}

Aucun

\section{Résumé}

Le mouvement «open access » $(\mathrm{OA})$ a eu notamment pour conséquence la mise en place de revues en libre accès, avec l'apparition, pour certaines d'entre elles, de frais de publication (Article Processing Charges) que doivent acquitter les auteurs pour publier. L'objectif de cette étude est double: - caractériser les articles produits par les chercheurs des établissements d'enseignement supérieur et de recherche orléanais sur l'année 2016 par rapport à l'OA (présence sur l'archive ouverte HAL, caractéristiques des revues dans lesquelles ont été publiés ces articles, APC qu'elles imposent théoriquement...), - comptabiliser les APC réellement payés par ces mêmes établissements. Les principaux résultats de cette étude mettent en évidence que le modèle hybride tend à s'imposer de plus en plus chez les éditeurs (pour 83,6\% des 923 articles étudiés, les auteurs avaient la possibilité de choisir l'option hybride). Concernant l'option hybride, même si - lorsqu'elle était proposée aux auteurs - elle a n'été choisie que dans $10 \%$ des cas, le nombre d'articles publiés selon cette option représentait 41,9\% des articles publiés en libre accès. Les dépenses d'APC prises en charge par la communauté orléanaise se répartissent uniquement sur 23 articles (sur 923 publiés), et la somme déboursée (39261 euros) est finalement assez limitée en regard des sommes totales dépensées pour les acquisitions des ressources documentaires par des institutions de taille comparable à celles de la communauté orléanaise. Les résultats présentés dans cette étude, offrent un élément de comparaison utile par rapport à des travaux antérieurs, permettant de mieux appréhender la problématique, complexe, de l'OA et des APC qui en découlent.

\section{Summary}

One of the corollaries of the open access movement (OA) has been the setting up of open access journals, with the occurrence, for some of them, of publication fees (Article Processing Charges) that authors must pay to publish. The aim of this study is twofold: - to characterize the articles produced by the researchers of the higher education and research institutions of Orléans (France) in 2016 in relation to the OA (presence on the open archive HAL, characteristics of the journals in which these articles have been published, APC they theoretically require...), - to record the actual APCs paid by these matching institutions. The 
main results of this study show that the hybrid model is becoming more and more popular among publishers (for $83.6 \%$ of the 923 articles studied, the authors had the possibility to choose the hybrid option). Regarding that hybrid option, even if it was offered to authors, it was only chosen in $10 \%$ of cases, the number of articles published under this option accounted for $41.9 \%$ of articles published in open access. The APC expenses borne by the Orleans community are spread over only 23 articles (out of 923 published), and the actual amount spent $(39,261$ euros) is in the end rather limited compared to the total amount spent for the acquisition of documentary resources by institutions of comparable size to those of the Orleans community. The results presented in this study offer a useful comparison with previous works, for a better understanding of the complex problematics of OA and its resulting APCs.

\section{Mots clés}

Libre accès ; publication ; revues ; frais de publication

\section{Keywords}

Open access ; publication ; journals ; article publication charges

\section{Rubrique}

Article original/Etudes 


\section{Introduction}

La polysémie de l'expression Open Access (OA) induit des notions multiples parfois sujettes à confusion. Si le concept de Green est relativement transparent (dépôt d'une version auteur non validée par les pairs ou pre-print sur une archive ouverte), celui de Gold ouvre la porte à plusieurs acceptions qui aboutissent toutes au même résultat, attendu par le chercheur : la levée des barrières commerciales à la consultation de sa production scientifique (mais pas à la production). Le Gold suppose par définition l'existence d'un modèle économique détenu à la source par les éditeurs commerciaux.

Si la liberté d'accès paraît naturellement essentielle pour accélérer la connaissance d'un domaine dans le cadre d'une recherche, cet aspect positif du Green reste parallèle par rapport aux usages d'évaluation : son accessibilité dans un format «final» et valorisant aux yeux des instances administratives d'évaluation. Le modèle d'évaluation bibliométrique standard génère en effet pour une part importante les critères adoptés par les classements internationaux des organismes, qui accordent une part notable à la production scientifique de recherche des établissements. Une publication remarquable est souvent associée conceptuellement, à tort, au facteur d'impact de la revue publiante et la présence dans des fichiers tels que la liste des Highly Cited Researchers, ou plus simplement le Web of Science. Une telle publication permet d'accroître la garantie de ressources pour un laboratoire et, fréquemment, l'accélération des évolutions de carrière. Suivant ce modèle, c'est l'article « officiel » publié dans une revue homologuée par des instances ou des outils du type que l'on vient de citer, qui fera foi pour l'évaluation administrative ; l'article apparaissant doté de la charte typographique d'un éditeur coté dans une revue estimée. Le preprint déposé suivant le modèle du Green, présente assez souvent des caractéristiques de contenu relativement proches du post-print. Il est toutefois normalement considéré comme un produit secondaire, marginal, dont on ne peut se targuer par exemple lors d'une campagne de recrutement. Il sera évidemment, en revanche, apprécié pour la qualité de son contenu et sa facilité d'accès ; mais il restera « au bord du chemin » pour l'évaluation finale, celle qui compte. Certes, depuis peu, le preprint acquiert quelques lettres de noblesse [1] et se voit cité dans des bibliographies terminales d'articles publiés dans des revues «agréées » ou «officielles». Toutefois, cette montée en puissance du preprint reste anecdotique et le modèle précité demeure prégnant.

Une fois établie la prééminence supposée de l'article paru dans la revue homologuée par les instances évaluatrices, il faut surmonter un autre obstacle, inhérent au modèle de la 
publication commerciale : l'accès par abonnement souvent doublé d'un embargo imposé par l'éditeur à une mise en ligne de type Green. En France, la loi Lemaire sur la République numérique a certes réduit ce délai d'un an à six mois en Sciences-Technologie-Médecine (STM), mais cela ne vaut que pour l'accès au post-print auteur («Fichiers produits par l'(les) auteur(s) » sur HAL), qui est une trace tangible de sa prestation, et pas à la «preuve » de la notabilité des auteurs, sanctionnée par le post-print éditeur homologué par la revue ellemême. Or c'est celle-ci qui conditionne le bon fonctionnement des institutions. Il faut donc que les articles « officiels » soient visibles immédiatement par la communauté pour garantir la réputation (et donc l'évolution en termes de performance) de l'entité productrice et de ses acteurs.

On le sait, les éditeurs ont depuis longtemps bien compris ce mécanisme. L'expression Open Access recouvre dès lors deux acceptions : le Full OA et l'Hybride.

Le Full $O A$ va lui-même se décomposer en quatre catégories :

- les revues délibérément en accès libre par nature et sans frais pour les lecteurs comme pour les auteurs. Ces journaux sont très peu nombreux et peuvent émaner directement de sociétés savantes ou d'organismes de recherche soucieux de valoriser leur production scientifique sans recourir à une logistique sophistiquée. Exemples: la Revista de Biología tropical de l'Université du Costa-Rica (ISSNs 0034-7744 et 2215-2075) ou le Journal of Microbiology, Biotechnology and Food Sciences JMBFS (ISSN 1338-5178) de la Faculté d'Agronomie de la République slovaque, deux journaux listés dans le Directory of Open Access Journals (DOAJ). À l'instar de nombreuses publications repérées dans le réservoir Scielo (http://www.scielo.org/php/index.php), les organismes situés dans les pays émergents communiquent le résultat de leurs investigations dans un «second cercle » en marge de ce que d'aucuns pourraient nommer (injustement sans doute) la «voie royale». - Cependant, des revues dotées de solides facteurs d'impact mais autrement payantes (abonnement / APC) peuvent aussi, occasionnellement, ouvrir certaines pages à des contributions ne nécessitant aucun paiement de la part des auteurs et bien sûr en accès libre pour les chercheurs. Par exemple, la revue Astronomy \& Astrophysics (ISSN 0004-6361) propose un accès libre aux sections Atomic, molecular, and nuclear data; Astronomical instrumentation; Catalogs and data et Numerical methods and codes incluses au sommaire de ses numéros, sans délai [2]. Là aussi, il s'agit d'un contingent très minoritaire de publications. 
- On peut également signaler les épirevues qui marient le concept du Green avec un peerreviewing classique. Elles sont naturellement exonérées de frais de la part du déposant en archive ouverte. Le CCSD propose quelques titres (exemple: Discrete Mathematics and Theoretical Computer Science (https://dmtcs.episciences.org/page/policies), bien sûr déposés sur HAL et ArXiv sous CC-BY 4.0)

- On ne peut nier que les exemples qui précèdent ne représentent qu'un nombre très modéré d'items dans le paysage des revues proprement dites de l'Open Access hors du champ Green. L'expression Full OA désignera souvent des journaux dont le principe implique les fameux frais de publication ou Article Processing Charges (APC), objet principal de la présente contribution. Pas d'abonnement, mais paiement à la source par les entités auteures. Dans le domaine STM, les publications en collaboration avec des chercheurs diversement issus de la communauté internationale peuvent être financées par l'une des équipes participantes, en tant que coauteure, plus à l'aise sur le plan budgétaire. Les personnes morales impliquées peuvent être aussi des fédérations d'organismes de recherche importantes. On peut citer le projet SCOAP3 (Sponsoring Consortium for Open Access Publishing in Particle Physics https://scoap3.org) du CERN qui cofinance en son sein le paiement des APC. Contrairement aux autres modèles Open Access où les bibliothèques, les auteurs ou les agences paient des frais pour chaque article, les éditeurs SCOAP3 ont un contrat unique avec le CERN qui paie de façon centralisée les APC. Les éditeurs éliminent ou réduisent à leur tour les coûts d'abonnement pour tous leurs «clients». Les partenaires de SCOAP3 redirigent les fonds précédemment utilisés pour payer les abonnements dans un pot commun, qui est ensuite utilisé pour les paiements centralisés des APC. Cela permet d'établir de manière compétitive un niveau d'APC acceptable tout en recyclant des fonds rendus disponibles. En avril 2018, 20 000 articles étaient ainsi proposés en OA par SCOAP3. Notons que ce modèle a été défendu à Paris au Ministère de l'Enseignement supérieur et de la Recherche par Denis Jérôme, membre de l'Académie des Sciences et professeur émérite de Physique à Paris-Sud, lors des Journées Open Access le 13 octobre 2015 , modèle qu'il jugeait opportun d'appliquer à l'ensemble du dispositif de publication en France [3].

Les revues Full OA avec APC bénéficient souvent de forts facteurs d'impact et s'acquitter d'une «taxe de publication » peut ne pas sembler inconsidéré à des coauteurs soucieux de leur performance, de leur carrière et du dynamisme de leur(s) entité(s) de recherche. Néanmoins, dans une étude actualisée en septembre 2016 [4], la Direction de l'Information scientifique et technique (DIST) du CNRS s'émeut des dépenses excessives (voire abusives) 
générées par les Article Processing Charges (APC) en publication scientifique. À ce sujet pourrait être évoqué le problème des revues prédatrices qui mettent en avant l'argument OA tout en imposant systématiquement des APC. La liste de Beall (https://beallslist.weebly.com), quel que soit son degré d'actualité, a le mérite de poser les bonnes questions et de générer une saine vigilance à ce sujet.

L'Hybride, parallèle au Full OA, génère ce fameux «double dipping » [5,6] qui au coût d'abonnement superpose celui d'APC. Même si le choix de l'option hybride est très décrié, il coexiste avec le Full OA dans des proportions qui ne cessent d'augmenter [7]. Cela pourrait signifier que, malgré le caractère on ne peut plus douteux sur le plan éthique de cette procédure éditoriale et commerciale, des entités de recherche privilégient l'objectif de la réputation du journal, même dans la perspective d'une soumission au mode hybride. Une réduction d'embargo à 6 mois en STM - par la loi Lemaire - est encore jugée trop insuffisante en regard de la nécessité d'apparaître rapidement et sans frais au lectorat et aux instances administratives évaluatrices dans la version « officielle » d'une revue homologuée parce que bien cotée, avec sa charte typographique. La pression à laquelle sont soumis les chercheurs devrait être atténuée par une prise de conscience plus volontariste des pouvoirs publics dans des négociations appuyées auprès des éditeurs (ce que fait le consortium Couperin avec une fermeté croissante et une conscience professionnelle reconnue) afin que des contraintes inhérentes au mode d'évaluation n'entravent pas l'égalité de tous dans un accès plus juste à la Science.

Les objectifs de cette étude sont les suivants : - caractériser les articles produits par les chercheurs des établissements d'enseignement supérieur et de recherche orléanais sur l'année 2016 par rapport à l'OA, en recensant leur présence sur l'archive ouverte HAL, tout en étudiant les caractéristiques des revues dans lesquelles ont été publiés ces articles (statut par rapport à l'OA et aux APC qu'elles imposent théoriquement), - comptabiliser les APC réellement payés par les établissements d'enseignement supérieur et de recherche orléanais sur cette même année 2016, - utiliser et tester une méthodologie basée sur le recueil «manuel » et non automatisé des données exploitées.

\section{Matériel et Méthodes}

La recherche des articles inclus dans cette étude a été effectuée en juillet 2017, en utilisant les bases de données bibliographiques Web of Science Core Collection (WoS) (http://isiknowledge.com/) et Scopus (https://www.scopus.com/Scopus.com). Afin de 
récupérer toutes les références d'articles produites par la communauté scientifique orléanaise, des requêtes spécifiques ont été élaborées en utilisant notamment les noms de laboratoires et codes associés (UMR...), ainsi que les toponymes de la région orléanaise. Les résultats ont été limités aux articles de recherche et revues de la littérature publiés en 2016. Le choix de la région orléanaise a été fait car celle-ci accueille une université pluridisciplinaire de taille moyenne, ainsi que des établissements de recherche divers (BRGM, INRA, CNRS...). Les références bibliographiques extraites du WoS et de Scopus ont été récupérées au format CSV et importées dans Microsoft Excel 2010 (® Microsoft, Redmond, USA) pour leur analyse. Avant celle-ci, un dédoublonnage des références a été réalisé en se basant sur le DOI et le titre des articles.

\section{Caractéristiques du corpus d’articles étudié}

Un examen des modalités de publication de la totalité des articles étudiés a été effectué dans les informations générales de l'éditeur ou de la revue, ou dans les recommandations aux auteurs. Pour chaque article a été déterminé le type de revue dans lequel il était publié en regard du libre accès aux articles : revue en libre accès total (Full OA), revue hybride sous abonnement proposant une option libre accès (Hybride), revue sous abonnement seul. L'accès au texte intégral de chaque article a été effectué sur les sites de leurs éditeurs respectifs, en utilisant les Digital Object Identifiers (DOI) à partir du site DOI.org. Afin de déterminer quels articles étaient en libre accès (c'est-à-dire ceux pour lesquels il n'est pas nécessaire de souscrire un abonnement), un accès informatique banalisé hors campus universitaire a été utilisé. Pour les articles en libre accès, la tarification des frais de publication a été recherchée sur le site de l'éditeur afin d'en évaluer le montant. Il faut préciser que les valeurs des frais de publication relevées sont «théoriques » et ne correspondent pas forcément aux sommes payées en réalité par les auteurs des articles. Ces données peuvent cependant fournir des indications sur les caractéristiques des frais de publication imposés globalement aux auteurs par les éditeurs. Lorsque les frais de publication étaient exprimés dans une autre devise que l'euro (Dollars, Francs suisses...), les sommes ont été converties en euros avec le taux de change en vigueur au 9 octobre 2017.

La présence ou l'absence de chaque article sur l'archive ouverte HAL (hal.archivesouvertes.fr) a également été évaluée, ainsi que la forme sous laquelle l'article apparaissait : présence de la référence bibliographique seule, présence du texte intégral sous forme «fichier auteur », présence du texte intégral sous forme «fichier éditeur » ou bien encore article sous embargo. 

communauté Orléanaise

Afin de déterminer les APC réellement engagés par les chercheurs de la communauté orléanaise, un e-mail a été envoyé à chaque auteur correspondant des articles déterminés comme étant en libre accès sur le site de leur éditeur. L'objectif étant d'interroger les auteurs sur l'existence ou non d'APC et de leur éventuel paiement, et dans l'affirmative de préciser quelle institution a supporté ces frais et quel en a été le montant exact. En l'absence de réponse de l'auteur correspondant, une recherche a été effectuée afin de trouver les coordonnées d'autres auteurs de l'article afin de les interroger sur le même sujet. Un total de 446 emails a ainsi été envoyé. En l'absence de réponses aux mails envoyés, Nathalie Potier a tenté localement d'entrer en contact avec les auteurs, les gestionnaires et les professionnels de l'Information Scientifique et technique Orléanais afin de recueillir ces informations.

\section{Résultats}

\section{Caractéristiques du corpus d'articles étudiés}

\section{Généralités}

La recherche des articles sur le WoS et Scopus a abouti à la découverte de 923 articles qui ont été publiés dans 522 journaux différents. La typologie des revues dans lesquelles ont été publiés ces 923 articles (Full OA, Hybride ou abonnement seul) est la suivante : 45 articles $(4,9 \%)$ ont été publiés dans des revues dont le contenu est consultable exclusivement sur abonnement, $106(11,5 \%)$ dans des revues «Full $O A »$ et $772(83,6 \%)$ dans des revues de type hybride.

Sur les 923 articles étudiés, 183 (soit 19,8\%) étaient accessibles en libre accès, dont 106 $(11,5 \%)$ provenaient de revues en Full OA, et 77 (8,3\%) de revues hybrides. Il faut noter que lorsqu'elle était proposée aux auteurs, l'option hybride a été choisie dans 10\% des cas (soit 77 articles sur 772).

\section{APC recueillis sur les sites des éditeurs}

Sur ces 183 articles en libre accès il a été possible de déterminer, à partir des données recueillies sur les sites des éditeurs, les APC théoriques pour 179 d'entre eux (Tableau 1), ainsi que leur répartition selon leur coût (Figure 1). 


\begin{tabular}{|c|c|c|l|l|l|l|}
\hline & $\begin{array}{l}\text { Nombre } \\
\text { d'articles } \\
(\%)\end{array}$ & $\begin{array}{l}\text { Coût total } \\
\text { des APC } \\
\text { théoriques } \\
(\%)\end{array}$ & $\begin{array}{l}\text { Coût } \\
\text { moyen des } \\
\text { APC } \\
\text { théoriques } \\
\text { par article }\end{array}$ & $\begin{array}{l}\text { Nombre } \\
\text { d'articles dont } \\
\text { les } \\
\text { théoriques sont } \\
\text { gratuits }\end{array}$ & $\begin{array}{l}\text { APC moyen par } \\
\text { article } \\
\text { considérer les APC } \\
\text { théoriques gratuits) }\end{array}$ \\
\hline $\begin{array}{l}\text { Articles } \\
\text { Full OA }\end{array}$ & $\begin{array}{c}104 \\
(58,1)\end{array}$ & $\begin{array}{c}130632 \\
(47,5)\end{array}$ & 1256,1 & 26 & 1674,8 \\
\hline $\begin{array}{l}\text { Articles } \\
\text { hybrides }\end{array}$ & $75(41,9)$ & $\begin{array}{c}144339 \\
(52,5)\end{array}$ & 1924,5 & 6 & 2091,9 \\
\hline Total & 179 & 274971 & 1536,1 & 32 & 1870,5 \\
\hline
\end{tabular}

Tableau 1: répartition et caractéristiques des articles en libre accès du corpus d'articles étudiés

Table 1: Distribution and characteristics of open access articles from the corpus of studied articles

Comme le montre le Tableau 1, la valeur moyenne théorique des APC des articles parus dans des revues Full OA est moins élevée que celle des articles parus dans des revues hybrides. De manière relativement surprenante, nous avons découvert des revues hybrides dont les APC étaient gratuits. Il s'agit par exemple de la revue Astronomy \& Astrophysics qui « offre» les APC lorsque les auteurs choisissent de publier leur article dans une catégorie thématique donnée (voir plus haut).

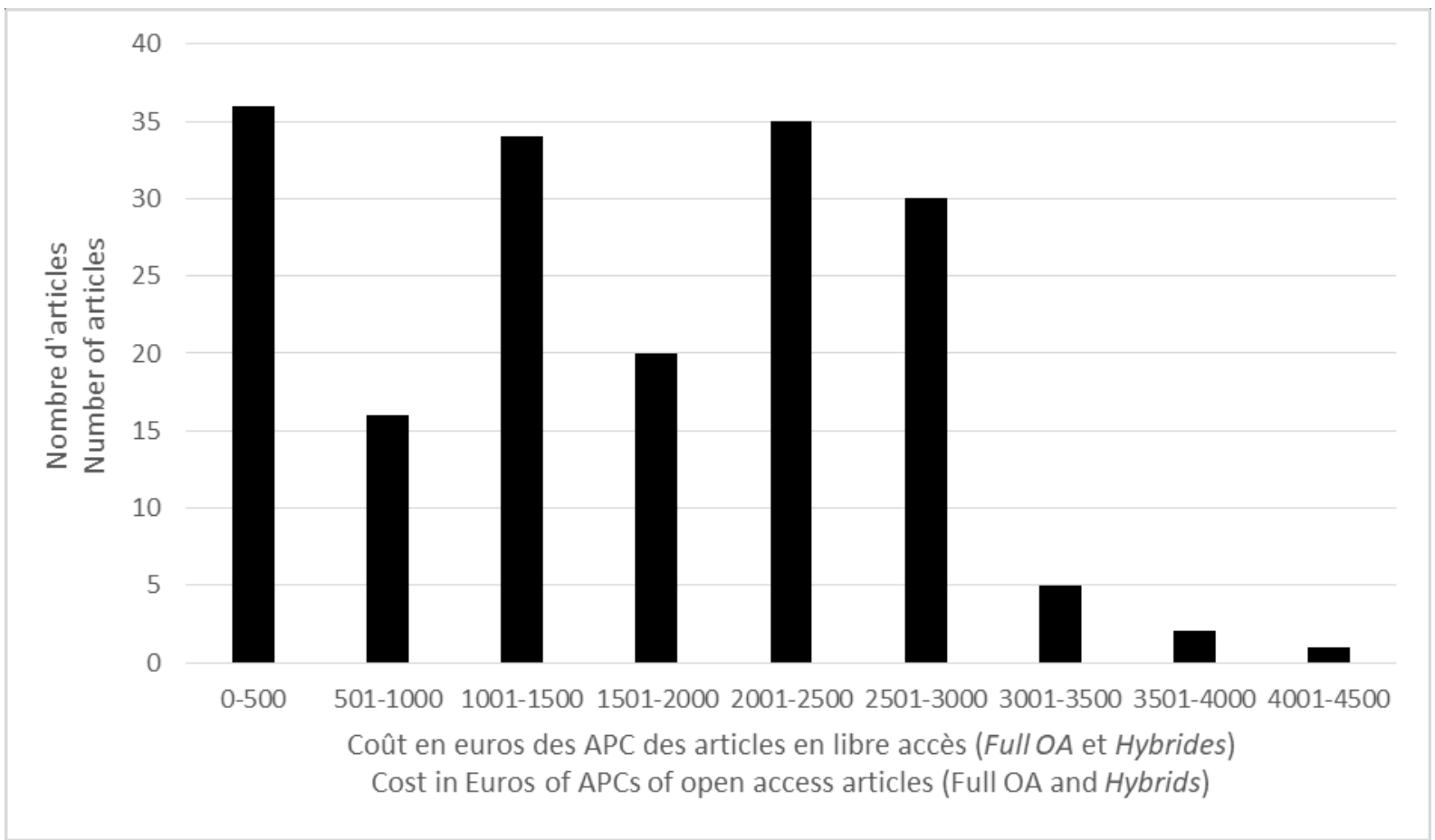

Figure 1. Répartition du coût des APC de la totalité des articles en libre accès (Full OA et Hybrides) Figure 1. Distribution of APC Costs of All Open Access articles (Full OA and Hybrids)

Comme le montre la Figure 1 les valeurs d'APC (Full OA et Hybrides) du corpus d'articles étudiés se répartissent sur une échelle comprise entre 0 et 4500 euros. Il faut noter l'existence 
d'un certain nombre d'APC gratuits (32, soit 17,9\%), et que les APC dont la valeur est supérieure à 3000 euros représentent finalement une fraction très faible des articles avec APC (8 articles, soit $4,6 \%)$.

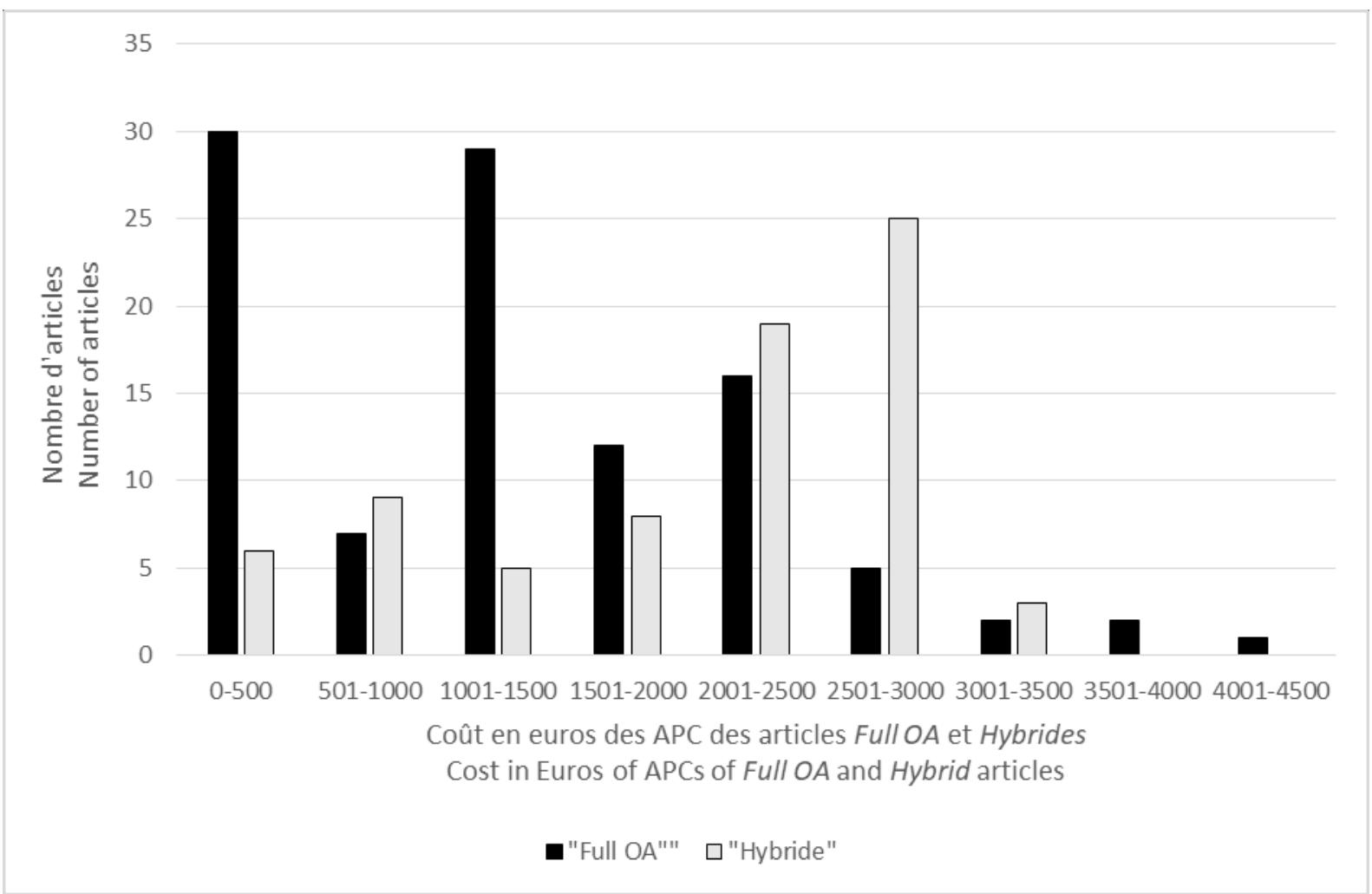

Figure 2. Répartition du coût des APC des articles en Full OA et Hybrides

Figure 2. Distribution of APC Costs of Full OA and Hybrid articles

Afin de pouvoir mieux appréhender les différences entre APC imposés par les revues Full OA et hybrides, la Figure 2 présente la répartition du coût des APC pour ces deux types de revues. Comme on peut le constater dans d'autres études antérieures (D. J. Solomon et Björk 2012 ; Morrison et al. 2015; Jahn et Tullney 2016), il existe un décalage entre les courbes Full OA et Hybrides, la courbe des APC hybrides étant globalement décalée d'une valeur approximative de 1000 euros supplémentaires. De plus le nombre d'articles Full OA ayant des APC de plus de 2500 euros est plutôt limité, tandis qu'il existe un nombre important d'APC provenant d'articles publiés dans des revues hybrides ayant des valeurs supérieures à 2500 euros. 


\begin{tabular}{|l|c|c|c|c|}
\hline Revue & $\begin{array}{l}\text { Type de la } \\
\text { revue "Full } \\
\text { OA"/Hybride }\end{array}$ & Coût total & $\begin{array}{l}\text { Nombre } \\
\text { d'articles }\end{array}$ & $\begin{array}{l}\text { Coût } \\
\text { moyen par } \\
\text { article }\end{array}$ \\
\hline Comptes Rendus Geoscience & Hybride & 28050 & 11 & 2550 \\
\hline Comptes Rendus Chimie & Hybride & 20400 & 8 & 2550 \\
\hline Scientific Reports & Full OA & 13050 & 10 & 1305 \\
\hline Atmospheric Chemistry And Physics & Full OA & 12941 & 6 & 2156,8 \\
\hline Plos One & Full OA & 12707,5 & 10 & 1270,8 \\
\hline Nature Communications & Full OA & 7400 & 2 & 3700 \\
\hline Chemistry Of Materials & Full OA & 6800 & 2 & 3400 \\
\hline European Journal Of Immunology & Hybride & 6000 & 2 & 3000 \\
\hline Oncotarget & Full OA & 5780 & 2 & 2890 \\
\hline Journal Of Experimental Botany & Hybride & 4800 & 2 & 2400 \\
\hline Astronomy \& Astrophysics & Hybride & 4760 & 13 & 366,2 \\
\hline Frontiers In Physics & Full OA & 4233 & 2 & 2116,5 \\
\hline Frontiers In Microbiology & Full OA & 4233 & 2 & 2116,5 \\
\hline Journal Of Chemical Physics & Hybride & 3740 & 2 & 1870 \\
\hline Inorganic Chemistry & Hybride & 3400 & 1 & 3400 \\
\hline Tuble
\end{tabular}

Tableau 2: Liste des 15 revues à l'origine des APC théoriques cumulés les plus élevés

Table 2: List of the 15 journals generating the highest aggregated theoretical APCs

Le Tableau 2 présente la liste des 15 revues dont les APC théoriques cumulés étaient les plus élevés. Il faut noter que parmi ces revues, 7 sur 15 sont de type hybride. Deux méga-journaux (Scientific Reports et PloS One) sont présents dans cette liste, confirmant l'attrait grandissant des chercheurs pour ces revues, déjà mis en évidence précédemment $[8,9]$.

\begin{tabular}{|l|r|r|l|r|r|}
\hline & Coût total & $\begin{array}{l}\text { Nombre } \\
\text { d'articles }\end{array}$ & $\begin{array}{l}\text { Coût } \\
\text { moyen } \\
\text { par } \\
\text { article }\end{array}$ & $\begin{array}{l}\text { loût } \\
\text { minimal }\end{array}$ & $\begin{array}{l}\text { Coût } \\
\text { maximal }\end{array}$ \\
\hline Editeur & 68255 & 31 & 2201,8 & 0 & 3060 \\
\hline Nature Publishing Group & 20450 & 12 & 1704,2 & 1305 & 3700 \\
\hline Copernicus GmbH & 19600 & 12 & 1633,3 & 105 & 4373 \\
\hline Frontiers Media SA & 19048,5 & 9 & 2116,5 & 2116,5 & 2116,5 \\
\hline Springer-Verlag & 17620 & 8 & 2202,5 & 2200 & 2220 \\
\hline Oxford University Press & 16937 & 8 & 2117,1 & 1300 & 2550 \\
\hline Public Library of Science & 14620 & 11 & 1329,1 & 1270,75 & 1912,5 \\
\hline Springer & 14300 & 9 & 1588,9 & 0 & 2525 \\
\hline American Chemical Society & 11900 & 4 & 2975 & 1700 & 3400 \\
\hline Mdpi Ag & 10716 & 7 & 1530,9 & 399 & 2052 \\
\hline Wiley-Blackwel & 8975 & 4 & 2243,8 & 0 & 3000 \\
\hline Impact Journals, Llc & 5780 & 2 & 2890 & 2890 & 2890 \\
\hline IOP Publishing Ltd & 5460 & 3 & 1820 & 1560 & 1950 \\
\hline Amer Inst Physics & 4887,5 & 3 & 1629,2 & 1147,5 & 1870 \\
\hline EDP Sciences & 4760 & 15 & 317,3 & 0 & 595 \\
\hline
\end{tabular}

Tableau 3 : Liste des 15 éditeurs à l'origine des APC théoriques cumulés les plus élevés

Table 3: List of the 15 publishers generating the highest aggregated theoretical APCs 
Le Tableau 3 montre que les APC peuvent varier de manière importante d'un éditeur à un autre et chez un même éditeur, comme cela a déjà été mis en évidence par [5,10-12]. À titre d'exemple, les valeurs d'APC se répartissent de 317,3 euros en moyenne pour EDP Sciences à 2975 euros pour l'American Chemical Society), et de 105 à 4373 euros chez Copernicus $\mathrm{GmbH}$.

\section{Autres caractéristiques du corpus d'articles}

Afin d'évaluer les dépôts réalisés sur HAL, nous avons identifié manuellement les articles présents sur HAL et la typologie du dépôt (sous embargo, texte auteur, post-print éditeur et référence). Comme le montre le Tableau 4, sur les 923 articles étudiés, 453 articles (49,1\%) ont été retrouvés sur HAL, dont 230 (24,9\%) en texte intégral (versions auteurs ou post-print éditeur). La proportion d'articles en OA ou non, présents sur HAL est tout à fait comparable (proche de 50\% dans les deux cas). De manière assez surprenante, près d'un quart $(23,7 \%$ ) des articles présents sur HAL le sont sous la forme d'une référence bibliographique sans que ne soit déposé le moindre texte intégral. Il faut également noter que, contre toute attente, 32 articles en OA ont été déposés sous la forme d'une référence bibliographique.

\begin{tabular}{|c|c|c|c|c|c|c|c|}
\hline & & & \multicolumn{4}{|c|}{ Type de dépôt } & \\
\hline & Non déposés & Déposés & $\begin{array}{l}\text { Sous } \\
\text { embargo }\end{array}$ & $\begin{array}{l}\text { Texte } \\
\text { auteur }\end{array}$ & $\begin{array}{l}\text { Post-print } \\
\text { éditeur }\end{array}$ & $\begin{array}{l}\text { Référence } \\
\text { (sans texte } \\
\text { intégral) }\end{array}$ & Total \\
\hline $\begin{array}{l}\text { Totalité des } \\
\text { articles }\end{array}$ & $470(50,9)$ & $453(49,1)$ & $4(0,4)$ & $111(12)$ & $119(12,8)$ & $219(23,7)$ & 923 \\
\hline $\begin{array}{l}\text { "Open } \\
\text { access » }\end{array}$ & $85(46,5)$ & $98(53,6)$ & $0(0)$ & $6(3,3)$ & $60(32,8)$ & $32(17,5)$ & 183 \\
\hline $\begin{array}{l}\text { Non " open } \\
\text { access" }\end{array}$ & 385 (52) & $355(48)$ & $4(0,5)$ & $105(14,2)$ & $59(8)$ & $187(25,3)$ & 740 \\
\hline
\end{tabular}

Etude des conditions réelles de l'existence et du paiement d'APC au sein de la communauté Orléanaise

Pour 162 articles sur les 183 en OA (soit 88,5\%), il a été possible de déterminer lesquels ont été à l'origine de paiement d'APC par un auteur ayant une affiliation dans une entité de recherche orléanaise. 23 articles ont ainsi fait l'objet d'une prise en charge d'APC, représentant une somme de 39261 euros, soit une moyenne de 1707 euros par article. Cette somme représente $14,3 \%$ de la totalité des APC théoriques que nous avons déterminés précédemment. Sur les 23 articles qui ont nécessité un paiement d'APC par une institution orléanaise, $18(78,3 \%)$ ont été publiés dans une revue Full OA et $5(21,7 \%)$ dans une revue 
hybride pour des coûts respectifs de 30337,5 euros et 8923,5 euros. Les coûts moyens des APC Full $O A$ et hybrides payés par la communauté orléanaise ont été respectivement de 1685,4 et 1784,6 euros

Les Tableaux 5 et 6 détaillent les 15 revues et les 10 éditeurs ayant entraîné les paiements d'APC les plus élevés par les auteurs orléanais. Parmi ces revues, seules 4 sur 15 sont des revues hybrides.

\begin{tabular}{|l|l|r|r|r|}
\hline Revue & $\begin{array}{l}\text { Type de la revue } \\
\text { "Full OA"/Hybride }\end{array}$ & $\begin{array}{l}\text { Coût } \\
\text { total }\end{array}$ & $\begin{array}{l}\text { Nombre } \\
\text { d'articles }\end{array}$ & $\begin{array}{l}\text { Coût } \\
\text { par article }\end{array}$ \\
\hline $\begin{array}{l}\text { Atmospheric Chemistry And } \\
\text { Physics }\end{array}$ & Full OA & 7886,3 & 3 & 2628,8 \\
\hline Scientific Reports & Full OA & 3830 & 3 & 1276,7 \\
\hline Nature Communications & Full OA & 3700 & 1 & 3700 \\
\hline $\begin{array}{l}\text { Atmospheric Measurement } \\
\text { Techniques }\end{array}$ & Full OA & 3034 & 2 & 1517 \\
\hline Cancer Letters & Hybride & 2554,5 & 1 & 2554,5 \\
\hline Continental Shelf Research & Hybride & 2554 & 1 & 2554 \\
\hline Geo-Marine Letters & Hybride & 2200 & 1 & 2200 \\
\hline $\begin{array}{l}\text { Microporous And } \\
\text { Mesoporous Materials }\end{array}$ & Full OA & 2125 & 1 & 2125 \\
\hline Frontiers In Marine Science & Full OA & 2116,5 & 1 & 2116,5 \\
\hline $\begin{array}{l}\text { Frontiers In Cellular } \\
\text { Neuroscience }\end{array}$ & Full OA & 1799 & 1 & 1799 \\
\hline Journal Of Cheminformatics & Full OA & 1465 & 1 & 1465 \\
\hline Solid Earth & Full OA & 1254 & 1 & 1254 \\
\hline $\begin{array}{l}\text { Journal Of Applied } \\
\text { Crystallography }\end{array}$ & Hybride & 1105 & 1 & 1105 \\
\hline Springerplus & Full OA & 1050 & 1 & 1050 \\
\hline Zookeys & Full OA & 888 & 1 & 888 \\
\hline
\end{tabular}

Tableau 5 : Liste des 15 revues ayant entraîné les paiements d'APC les plus élevés

Table 5: List of the 15 journals generating the highest APCs 


\begin{tabular}{|l|r|r|}
\hline Editeur & $\begin{array}{l}\text { Nombre } \\
\text { d'articles }\end{array}$ & Coût total \\
\hline Copernicus Gmbh & 6 & 12174,3 \\
\hline $\begin{array}{l}\text { Nature Publishing } \\
\text { Group }\end{array}$ & 4 & 7530 \\
\hline Elsevier & 3 & 7233,48 \\
\hline Frontiers Media Sa & 3 & 4595,525 \\
\hline Biomed Central Ltd & 2 & 2515 \\
\hline Springer Verlag & 1 & 2200 \\
\hline $\begin{array}{l}\text { Int Union } \\
\text { Crystallography }\end{array}$ & 1 & 1105 \\
\hline Pensoft Publ & 1 & 888 \\
\hline $\begin{array}{l}\text { European Assoc } \\
\text { Geoscientists \& } \\
\text { Engineers }\end{array}$ & 1 & 510 \\
\hline $\begin{array}{l}\text { Hindawi Publishing } \\
\text { Corporation }\end{array}$ & 1 & 510 \\
\hline
\end{tabular}

Tableau 6: Liste des 10 éditeurs ayant entraîné les paiements d'APC les plus élevés Table 6: List of the 10 publishers generating the highest APCs

Comparaison de différentes méthodologies couramment employées pour attribuer les APC aux différentes institutions de recherche

L'attribution du paiement des APC à la communauté orléanaise a été réalisée en interrogeant les auteurs de tous les articles en libre accès. Nous avons voulu comparer ces données à celles obtenues par deux méthodes couramment utilisées dans la littérature pour attribuer le paiement des APC aux organismes de recherche. La première consiste à considérer l'adresse $\mathrm{du}$ «corresponding author» pour attribuer l'origine du paiement des APC, tandis que la seconde considère l'adresse du premier ou du dernier auteur pour effectuer cette attribution. Dans le premier cas le nombre d'articles dont le paiement aurait été attribué à la communauté orléanaise aurait été de 62, avec un total d'APC payés de 88887,5 euros. Dans le deuxième cas, ce nombre aurait été de 87 avec un total d'APC payés de 113757,2 euros. Il faut noter que ces 2 méthodes aboutissent à des chiffres bien supérieurs à ceux que nous avons déterminés par notre méthode «manuelle », et donc à une surestimation des APC payés, comme cela avait déjà été mis en évidence auparavant [13].

\section{Discussion}

Les difficultés pour évaluer les dépenses liées aux APC dans les institutions de recherche ont été relevées par un certain nombre d'auteurs [5] et sont principalement dues : 
- à l'existence d'accords entre certaines institutions et éditeurs pour payer de manière globale et en amont les APC pour certaines revues, permettant aux auteurs de ces institutions de bénéficier d'APC réduits ou même de la gratuité lors de la publication,

- au manque de transparence concernant la diffusion et l'accès à ces données [10],

- à la difficulté d'attribuer le paiement des APC à un organisme de recherche donné dans les cas où les articles ont des auteurs appartenant à de organismes différents [13],

- à la difficulté de répertorier les articles publiés en open access hybrides et les APC qui en découlent [11,14],

- et enfin à l'imprécision d'un certain nombre de sites répertoriant les revues en libre accès (DOAJ...) et les montants des APC [13-15].

Malgré ces difficultés, certaines études étrangères ont tenté d'analyser les APC des revues en libre accès et d'évaluer les dépenses d'APC à partir des dépenses réalisées par les institutions en les interrogeant, afin que celles-ci leur fournissent les données comptables de ces frais de publication [5,16]. En France, cette option a été adoptée par le consortium Couperin dans les investigations qu'il a diligentées en 2017, seconde vague d'une enquête initiée par lui en 2015. Les données ainsi recueillies rejoignent un projet international coordonné par l'université de Bielefeld: Open APC [17]. D'autres études ont également tenté de déterminer les APC à partir de données obtenues à partir d'une ou plusieurs sources [10,12,18]. L'objectif de la présente étude était d'utiliser une méthodologie différente de ces études permettant théoriquement de contourner les difficultés précédemment évoquées. Cette méthodologie, basée sur le recueil et l'analyse «manuels» des données, sans aucun traitement automatique, permet d'obtenir des données validées et robustes, notamment concernant la détermination du statut des articles vis-à-vis de 1'OA, la détermination des APC théoriques sur le site des éditeurs (nous avons ainsi pu déterminer les APC théoriques pour 179 des 183 articles que nous avons étiquetés comme étant en OA). L'interrogation directe des auteurs a permis également de déterminer très précisément l'origine et le montant des APC réellement payés d'une grande fraction des articles en OA (pour 162 des 183 des articles).

Notre étude comporte cependant des limitations. Les résultats obtenus concernent la communauté de recherche orléanaise qui n'est pas forcement représentative de toutes les communautés de recherche françaises, ce qui implique que nos résultats ne sont pas généralisables. Néanmoins cette communauté associe des organismes pluridisciplinaires, des 
établissements publics à caractère scientifique et technologique (EPST) et une université, offrant ainsi une diversité intéressante. Concernant les valeurs des APC, nous n'avons pas pris en compte les éventuels frais de soumission, de reviewing, de mise en page (frais supplémentaires pour les figures en couleurs ou pages complémentaires) et de gestion associés au versement des APC par les institutions [5]. Notre méthodologie, qui utilise les bases de données WoS et Scopus, ne nous a pas permis de détecter et inclure les articles publiés chez des éditeurs prédateurs [19]. L'étude d'une seule année de publication (2016) n'a pas pu mettre en évidence de tendance concernant le nombre d'APC payés et leur montant, comme ont pu le faire d'autres études [16]. Enfin, il nous faut reconnaître que la lourdeur des opérations à mener et leur aspect chronophage (qui a déjà été souligné par [11]) pour aboutir aux présents résultats, interdit d'envisager d'appliquer cette méthodologie à un nombre très important d'articles. En effet, il semble peu raisonnable de vouloir appliquer notre méthodologie à des corpus de plus de 1000 articles.

Un nombre conséquent d'études a montré que la fraction d'articles disponibles en OA ne cesse de croître et s'établit actuellement aux alentours de 30 à $50 \%$ (ces chiffres sont très dépendants à la fois de la discipline et des méthodologies employées pour les déterminer) [20]. Le chiffre que nous avons relevé sur le corpus que nous avons étudié est très inférieur $(19,8 \%)$, car il ne tient compte que des articles publiés selon la voie dorée, sans tenir compte de la voie verte. En ce qui concerne les valeurs d'APC pratiquées par les revues en OA, celles-ci semblent vouloir imposer des APC de plus en plus importants au fur et à mesure du temps : de moins de 1000 \$ en moyenne en 2002 [18], à un peu plus de 1200 \$ en 2015 [21]. Il faut noter que nos résultats s'inscrivent bien dans cette tendance, puisque les valeurs d'APC théoriques du corpus d'articles que nous avons étudiés étaient de près de $1700 \$$ en moyenne. Comme l'ont montré des études antérieures [7,22,23], le modèle hybride tend à s'imposer de plus en plus chez les éditeurs. Nos résultats confirment ce phénomène, car pour $83,6 \%$ des articles étudiés, les auteurs avaient la possibilité de choisir l'option hybride. Les éditeurs commerciaux semblent donc s'être très largement orientés vers ce type de revue qui cumule abonnement et APC : le fameux «double dipping », très largement et de plus en plus décrié par les institutions de recherche. Cet engouement est concomitant, comme nous l'avons montré, au fait que les valeurs d'APC des revues hybrides sont plus élevées que celle des revues Full $O A$ (ce phénomène a déjà été mis en évidence précédemment dans la littérature $[5,10,12,14,24])$. Malgré ce surcoût, les auteurs semblent de plus en plus attirés par ce modèle, le nombre d'articles publiés en OA hybride étant passé selon [7] de 8000 en 2009 à 
45000 en 2016, avec une accélération de cette augmentation marquée à partir de l'année 2014. Dans la présente étude, même si cette option a été choisie par les auteurs uniquement dans $10 \%$ des cas où elle leur était proposée (77 fois sur 772), le nombre d'articles publiés dans une revue OA hybride représente 41,9\% des articles publiés en libre accès, une proportion nettement plus importante que celle mise en évidence par des études antérieures : de 1 à $2 \%$ en 2011 [22], et de 5,2\% pour l'année 2013 [23]. Afin de limiter le recours à ce modèle peu vertueux, il est tout à fait probable que l'on se dirige, a l'instar de l'INRIA, vers une interdiction quasi généralisée de publier en choisissant l'option hybride (et donc de payer des APC) dans ces revues, même si l'application du Plan S en 2020 risque potentiellement d'entretenir et favoriser ce modèle [25].

Parmi les motivations pour publier en OA, apparaît en bonne place la volonté des auteurs de mettre à disposition rapidement leur travail, notamment via une archive ouverte afin d'en augmenter la visibilité et donc potentiellement les citations qui en seront faites par leurs collègues [26-28]. Dans ce contexte, il est assez étonnant de constater que la proportion d'articles en OA présents sur HAL est tout à fait comparable à celle des articles sur abonnement (proche de 50\% dans les deux cas), et que les auteurs choisissant de publier en OA afin, entres autres d'augmenter la visibilité de leur article, n'utilisent pas plus l'archive ouverte HAL pour promouvoir leur production scientifique auprès de leur collègues. Nous avons été également surpris du fait que près de $25 \%$ des articles déposés sur HAL le soient sous forme de références bibliographiques sans texte intégral, montrant ainsi une déviation de l'objectif premier de HAL, qui était de favoriser l'accès aux textes intégraux des articles. Enfin, de manière encore plus étonnante, 32 articles publiés en OA étaient présents sur HAL sous forme d'une référence bibliographique sans texte intégral, sans que l'on puisse en expliquer les raisons de manière évidente (manque d'information/formation sur l'OA et les archives ouvertes de ces chercheurs?).

Concernant les articles dont les APC ont été pris en charge par la communauté orléanaise, ils ont représenté 23 articles sur les 162 qui ont été publiés en OA (soient 14,2\%). Ce nombre, ainsi que la somme déboursée (39261 euros) sont finalement assez limités en regard du nombre total d'articles publiés (923) et des sommes en jeu, par comparaison avec les sommes totales dépensées pour les acquisitions des ressources documentaires par les institutions de taille comparable à celles de la communauté orléanaise [29]. La valeur moyenne des APC payés par la communauté orléanaise (1707 euros par article) est un peu plus élevée que celle trouvée par [5] dans 23 universités au Royaume-Uni pour l'année 2014 (1435 euros), et de 
celle relevée par [10] pour 30 universités allemandes (1427 euros) sur la période 2005-2015. Elle est en revanche tout à fait en adéquation avec les données disponibles en novembre 2018 sur le site Open@APC (https://treemaps.intact-project.org), concernant 23 universités françaises (1604 euros en moyenne par APC payés sur l'année 2015). Remarquons qu'en ces temps de contraintes budgétaires avérées dans les institutions de recherche française, cette valeur moyenne de 1707 euros est loin d'être anecdotique lorsque celle-ci doit être prise en charge directement par une institution de recherche ou une unité de recherche.

Nous avons mis en évidence une différence notable de proportion entre la quantité et les valeurs d'APC théoriques et celles réellement prises en charge par la communauté orléanaise. Le pourcentage d'articles publiés dans des revues hybrides et la fraction des sommes engagées pour cela sont ainsi près de deux fois moins importants que les valeurs correspondantes mises en évidence théoriquement pour la totalité des articles. Sans que l'on puisse l'affirmer ou l'infirmer, une des hypothèses que l'on peut émettre afin de tenter d'expliquer ces différences pourrait être que les auteurs orléanais sont moins enclins que leurs collègues avec qui ils collaborent à publier dans des revues hybrides et à payer des APC pour ces revues. La plus faible proportion de revues de type hybride parmi les 15 revues ayant entraîné le paiement d'APC les plus élevés par les auteurs orléanais (Tableau 5), par rapport aux valeurs globales théoriques de la totalité du corpus (Tableau 2), semble confirmer cette hypothèse. Ou bien encore, autre hypothèse pouvant expliquer ces différences, l'existence d'accords en amont entre éditeurs et institutions, permettant aux auteurs orléanais de publier gratuitement ou de bénéficier d'APC réduits dans certaines revues hybrides. Toutefois, la proportion d'articles provenant de paiement d'APC hybrides que nous avons fait ressortir $(21,7 \%)$ est du même ordre de grandeur que les 27,1\% mis en évidence [11] pour les université d'Aix-Marseille et de Lorraine (58 articles sur 214 pour les années 2013 à 2015), et les $14,5 \%$ de l'étude «Couperin » pour les 21 universités dont les résultats sont disponibles sur le site Open@APC pour l'année 2015 (217 articles sur 1498, soit 14,5 \%), et ceci malgré des méthodologies déployées très différentes de celle utilisée dans la présente étude. Les coûts moyens des APC Full $O A$ et hybrides payés par la communauté orléanaise ont été respectivement de 1685,4 et 1784,6 euros. À noter que la différence entre APC Full OA et hybrides réellement payés est moins importante que celle retrouvée par [11] (897,8 euros), par [5] (787,8 euros) et par l'étude Couperin sur l'année 2015 (620 euros), sans que nous soyons en mesure de l'expliquer. 
Considérant un corpus de références, les méthodes visant à imputer les dépenses d'APC à une institution plutôt qu'à une autre sont, on l'a vu, naturellement génératrices de résultats d'analyse relativement fluctuants. En conséquence, en dépit de cette réserve de principe et compte tenu de leur caractère malgré tout significatif, les résultats présentés dans cette étude ont sans doute le mérite d'offrir un élément de comparaison utile par rapport à des travaux identiques, afin de mieux appréhender la problématique, complexe, de l'OA et des APC qui en découlent. Les résultats de cette étude peuvent également permettre de sensibiliser les chercheurs, enseignants/chercheurs et doctorants à ces nouveaux modèles éditoriaux et de publication. Incidemment, cette même étude a d'ailleurs donné lieu à une conférence à l'Observatoire des Sciences de l'Univers de la région Centre (OSUC-CNRS), à Orléans le 21 novembre 2017 à l'initiative de ses trois auteurs, pour sensibiliser les chercheurs, les professionnels de l'IST et les gestionnaires de la communauté scientifique orléanaise, à l'impact des APC.

\section{Remerciements}

Ce projet de recherche a reçu un financement spécifique du GIS du Réseau des Urfist.

\section{Déclaration de liens d’intérêts}

Les auteurs déclarent ne pas avoir de liens d'intérêts.

\section{Références}

1. The Stars Are Aligning for Preprints [Internet]. The Scholarly Kitchen. 2017 [cite 20 nov 2018]. Disponible sur: https://scholarlykitchen.sspnet.org/2017/04/18/stars-aligningpreprints/

2. Astronomy \& Astrophysics (A\&A) [Internet]. [cité 20 nov 2018]. Disponible sur: https://www.aanda.org/about-aa/open-access-policy

3. Les 6èmes journées Open Access - Denis Jerôme [Internet]. [cité 20 nov 2018]. Disponible sur: https://www.canalu.tv/video/fmsh/les_6emes_journees_open_access_denis_jerome.19602

4. DIST CNRS. Financer la publication scientifique. Le «Lecteur» et / ou «l'Auteur»? Evolutions, Alternatives. Observations de la DIST [Internet]. 2016 sept p. 21. Disponible sur: http://www.cnrs.fr/dist/z-outils/documents/Distinfo2/DISTetude3_09.2016-final.pdf

5. Pinfield S, Salter J, Bath PA. The "total cost of publication" in a hybrid open-access environment: Institutional approaches to funding journal article-processing charges in combination with subscriptions. J Assoc Inf Sci Technol. 1 juill 2016;67(7):1751- 66. 
6. Horava T, Ward M. Library Consortia and Article Processing Charges: An International Survey. Ser Rev. oct 2016;42(4):280- 92.

7. Björk B-C. Growth of hybrid open access, 2009-2016. PeerJ. 29 sept 2017;5:e3878.

8. Björk B-C. Have the "mega-journals" reached the limits to growth? PeerJ. 2015;3:e981.

9. Wakeling S, Willett P, Creaser C, Fry J, Pinfield S, Spezi V. Open-Access MegaJournals: A Bibliometric Profile. PLOS ONE. 18 nov 2016;11(11):e0165359.

10. Jahn N, Tullney M. A study of institutional spending on open access publication fees in Germany. PeerJ. 9 août 2016;4:e2323.

11. Delhaye M, Lutz J-F. Mesurer les dépenses d'APC: méthodologie et étude de cas. Approche comparée Aix Marseille Université - Université de Lorraine. Rev Fr Sci L'information Commun. 2017;11.

12. Solomon D, Björk B-C. Article processing charges for open access publication-the situation for research intensive universities in the USA and Canada. PeerJ. 21 juill 2016;4:e2264.

13. Bonvallot V, Chrétien S, Badolato A-M. Publications françaises Open Access 20102014 - Etude bibliométrique [Internet]. Inist-CNRS; BSN4; 2017 févr [cité 20 mars 2017]. Disponible sur: https://archivesic.ccsd.cnrs.fr/sic_01472799/document

14. Morrison H, Salhab J, Calvé-Genest A, Horava T. Open Access Article Processing Charges: DOAJ Survey May 2014. Publications. 5 févr 2015;3(1):1- 16.

15. Arouerghi. Comparaison DOAJ et MDPI: Titres et APC [Internet]. Sustaining the Knowledge Commons / Soutenir les savoirs communs. 2017 [cité 18 mai 2017]. Disponible sur: https://sustainingknowledgecommons.org/2017/03/02/comparaison-doajet-mdpi-titres-et-apc/

16. Pinfield S, Middleton C. Researchers' Adoption of an Institutional Central Fund for Open-Access Article-Processing Charges: A Case Study Using Innovation Diffusion Theory. SAGE Open. 25 janv 2016;6(1):2158244015625447.

17. OpenAPC - Open APC [Internet]. [cité 18 mai 2017]. Disponible sur: https://treemaps.intact-project.org/apcdata/openapc/

18. Solomon DJ, Björk B-C. A study of open access journals using article processing charges. J Am Soc Inf Sci Technol. 1 août 2012;63(8):1485- 95.

19. Perlin MS, Imasato T, Borenstein D. Is predatory publishing a real threat? Evidence from a large database study. Scientometrics. juill 2018;116(1):255- 73.

20. Boudry C, Durand-Barthez M. [Open access in biology and medicine: Historical review and state of the art in 2016]. Ethics Med Public Health. 2017;3(1):168- 80.

21. Björk B-C, Solomon D. Article processing charges in OA journals: relationship between price and quality. Scientometrics. 13 mars 2015;103(2):373- 85. 
22. Björk B-C. The hybrid model for open access publication of scholarly articles: A failed experiment? J Am Soc Inf Sci Technol. 1 août 2012;63(8):1496- 504.

23. Laakso M, Björk B-C. Hybrid open access-A longitudinal study. J Informetr. nov 2016;10(4):919- 32.

24. Van Noorden R. Open access: The true cost of science publishing. Nature. 2013;495:426- 9 .

25. Science Europe - cOAlition S [Internet]. [cité 5 oct 2018]. Disponible sur: https://www.scienceeurope.org/coalition-s/

26. Craig ID, Plume AM, McVeigh ME, Pringle J, Amin M. Do open access articles have greater citation impact?: A critical review of the literature. J Informetr. juill 2007;1(3):239- 48.

27. Tennant JP, Waldner F, Jacques DC, Masuzzo P, Collister LB, Hartgerink CHJ. The academic, economic and societal impacts of Open Access: an evidence-based review. F1000Research. 2016;5:632.

28. Nelson GM, Eggett DL. Citations, mandates, and money: Author motivations to publish in chemistry hybrid open access journals. J Assoc Inf Sci Technol. oct 2017;68(10):2501- 10 .

29. Les dépenses documentaires des universités [Internet]. Ministère de l'Enseignement supérieur, de la Recherche et de l'Innovation. [cité 19 nov 2018]. Disponible sur: //www.enseignementsup-recherche.gouv.fr/cid106569/les-depenses-documentaires-desuniversites.html 